\title{
VECTOR MESON PRODUCTION AND NUCLEON RESONANCE ANALYSIS IN THE GIESSEN COUPLED-CHANNEL MODEL
}

\author{
G. PENNER ${ }^{*}$ AND U. MOSEL \\ Institut für Theoretische Physik, Universität Giessen, D-35392 Giessen \\ *E-mail: gregor.penner@theo.physik.uni-giessen.de
}

\begin{abstract}
We present a nucleon resonance analysis by simultaneously considering all pionand photon-induced experimental data on the final states $\gamma N, \pi N, 2 \pi N, \eta N, K \Lambda$, $K \Sigma$, and $\omega N$ for energies from the nucleon mass up to $\sqrt{s}=2 \mathrm{GeV}$. In this analysis we find strong evidence for various higher lying resonances as, e.g., the $P_{13}(1900)$. The $\omega N$ production mechanism is dominated by large $P_{11}(1710)$ and $P_{13}(1900)$ contributions.
\end{abstract}

\section{Introduction}

The reliable extraction of nucleon resonance properties from experiments where the nucleon is excited via either hadronic or electromagnetic probes is one of the major issues of hadron physics. The goal is to be finally able to compare the extracted masses and partial-decay widths to predictions from lattice QCD and/or quark models (see references in Refs. ${ }^{1,2,3}$ ).

Basically all information about nucleon resonances identified so far from experiment stems from analyses of pion-induced $\pi N$ and $2 \pi N$ production and from pion photoproduction, see ${ }^{4}$ and references therein. However, it is well known that, for example, in the case of the $S_{11}(1535)$ the consideration of the $\eta N$ final state is inevitable to extract its properties reliably, and similar effects can be expected for higher-lying resonances and different thresholds. On the other side, quark models predict a much richer resonance spectrum than has been found in $\pi N$ and $2 \pi N$ production so far, giving rise to speculations that many of these resonance states only couple weakly to the $\pi N$ final state ("missing" resonances). For a consistent and reliable identification of these resonances and their properties, the consideration of unitarity effects are inevitable and as many final states as possible have to be taken into account simultaneously. With this aim in mind we have developed in Refs. ${ }^{1,2,5,6}$ a unitary coupled-channel effective Lagrangian model 
(Giessen model) that incorporates the final states $\gamma N, \pi N, 2 \pi N, \eta N, K \Lambda$, $K \Sigma$, and in particular $\omega N$. This model is used for a simultaneous analysis of all available experimental data on photon- and pion-induced reactions on the nucleon in the energy range $m_{N}<\sqrt{s}<2 \mathrm{GeV}$. The premise is to use the same Lagrangians for the pion- and photon-induced reactions allowing for a consistent analysis, thereby generating the background dynamically from Born, $u$ - and $t$-channel contributions without new parameters.

Here, we only point out some aspects of our results; all the details can be found in Refs. ${ }^{1,2,3}$.

\section{The Giessen Model}

In the Giessen model the Bethe-Salpeter equation for the scattering amplitude $\mathcal{T}$ is solved by the $K$-matrix approximation, where the intermediate particles are set on their mass shell. By a partial-wave decomposition in total spin $J$, parity $P$, and isospin $I$, which is performed in a generalized and uniform way for all final-state combinations (see Refs. ${ }^{1,2,3}$ for details), one arrives at an algebraic equation relating the decomposed $\mathcal{T}_{f i}$ and the potential $\mathcal{V}_{f i}$ (in a schematic notation): $\mathcal{T}_{f i}^{I J \pm}=\left[\mathcal{V}^{I J \pm} /\left(1-\mathrm{i} \mathcal{V}^{I J \pm}\right)\right]_{f i}$. Hence unitarity is fulfilled as long as $\mathcal{V}$ is Hermitian. Furthermore, in the effective Lagrangian approach generating the potential $\mathcal{V}$, gauge invariance is fulfilled in a straightforward way ${ }^{2,3}$.

The nucleon resonance properties are extracted by a $\chi^{2}$ minimization procedure using a total of about 6800 experimental data points, comprising partial-wave analyses for $\gamma / \pi N \rightarrow \pi N, \pi N \rightarrow 2 \pi N$, and total and differential cross section and polarization data for all other reactions (see Refs. $\left.{ }^{1,2,3}\right)$; a good simultaneuos description of all channels is achieved. As examples, we show the resulting total cross sections of $\eta N, K \Lambda$, and $\omega N$ production in Fig. 1. In these reactions and in particular in $\omega N$ production, strong evidence for important $P$-wave contributions is found, mainly steming from the $P_{11}(1710)$ and $P_{13}(1900)$ resonances, where the latter one is especially important in the pion-induced reactions. In $K \Lambda$ photoproduction also a clear threshold effect is visible due to the opening of the $\omega N$ final state. Note that in $\omega N$ photoproduction, we furthermore observe a large contribution of the $\pi t$-channel exchange. In Fig. 2, the $\pi N$ inelastic partial-wave cross sections for $I=\frac{1}{2}$ are shown, emphasizing the importance of the inclusion of final states apart from $\pi N$ and $2 \pi N$ in a unitary model. In the $J^{P}=\frac{1}{2}^{-}\left(S_{11}\right), \frac{1}{2}^{+}\left(P_{11}\right)$, and $\frac{3}{2}^{+}\left(P_{13}\right)$ partial waves the consideration of the $\eta N$ and $\omega N$ final states are particularly important. 

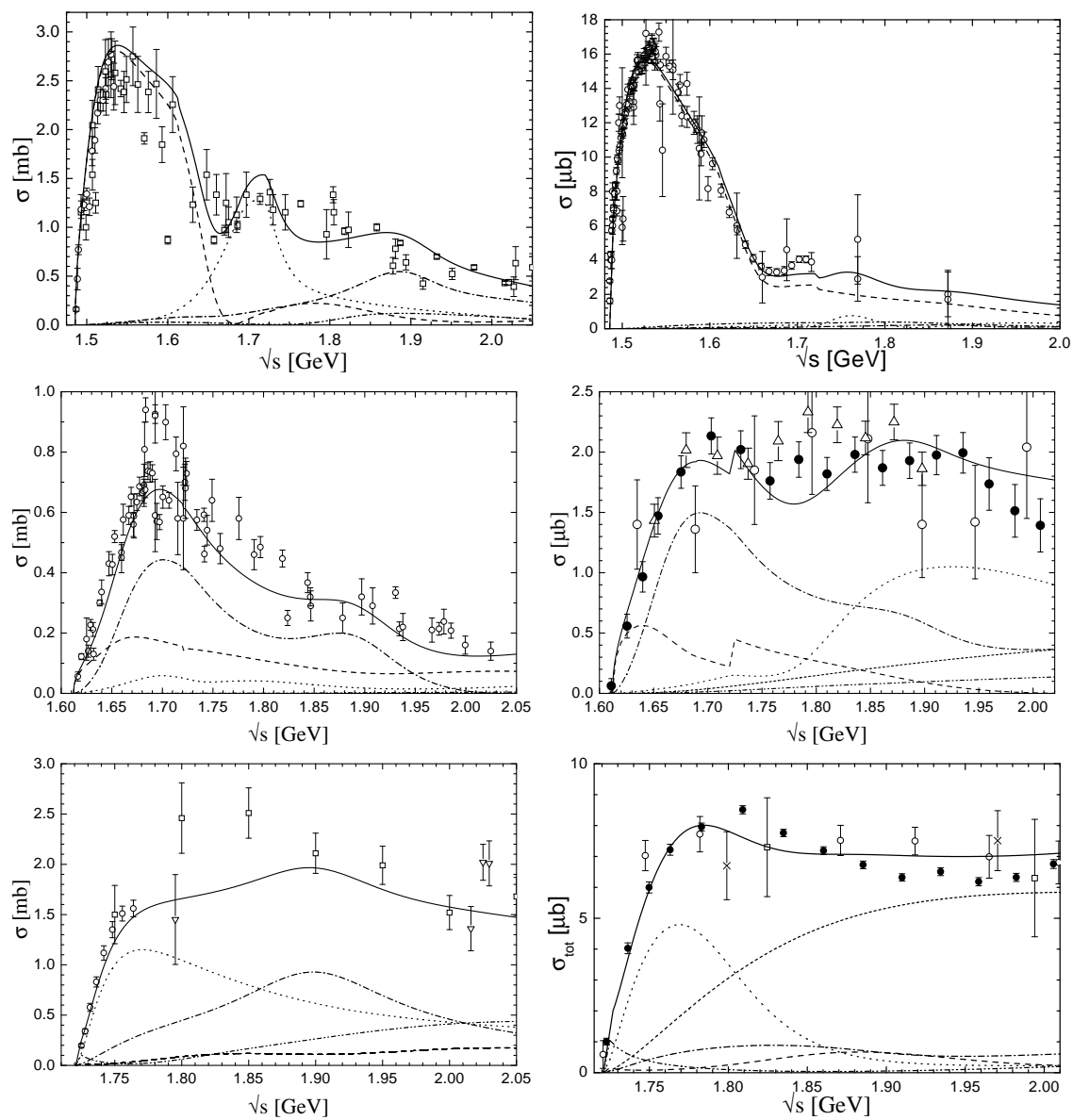

Figure 1. Examples of total cross section results. Upper left: $\pi^{-} p \rightarrow \eta n$, upper right: $\gamma p \rightarrow \eta p$, middle left: $\pi^{-} p \rightarrow K^{0} \Lambda$, middle right: $\gamma p \rightarrow K^{+} \Lambda$, lower left: $\pi^{-} p \rightarrow$ $\omega n$, lower right: $\gamma p \rightarrow \omega p$. Partial-wave contributions: $J^{P}=\frac{1}{2}^{-}\left(S_{11}\right)$ : dashed line; $\frac{1}{2}^{+}\left(P_{11}\right)$ : dotted line; $\frac{3}{2}^{+}\left(P_{13}\right)$ : dash-dotted line; $\frac{3}{2}^{-}\left(D_{13}\right)$ : dash-double-dotted line (in brackets the $\pi N$ notation is given). The sum of all partial waves is given by the solid line. For $K \Lambda$ and $\omega N$ photoproduction, also the contribution of higher partial waves $\left(J \geq \frac{5}{2}\right)$ is indicated by the short-dashed line. For the data references, see Refs. ${ }^{1,2,3}$.

\section{Outlook}

The next step towards a further improvement of the model is the inclusion of spin- $\frac{5}{2}$-resonance effects, see Ref. ${ }^{7}$. Furthermore, investigations concerning a detailed inclusion of all individual $2 \pi N$ final states $(\rho N, \pi \Delta, \ldots)$ are in 


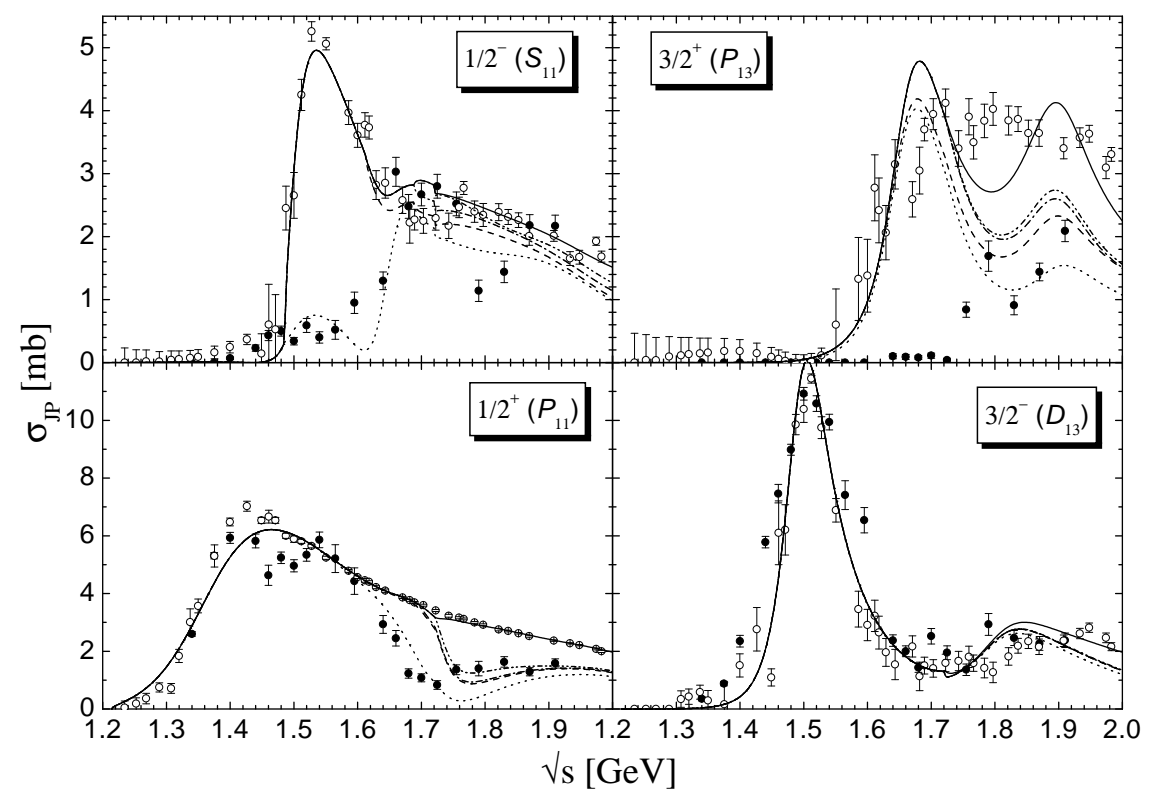

Figure 2. Decomposition of the inelastic partial-wave cross sections of $\pi N \rightarrow \pi N$ for isospin $\frac{1}{2}$. Partial-wave cross section of $2 \pi N$ : dotted, $+\eta N$ : dashed, $+K \Lambda$ : dash-dotted, $+K \Sigma$ : dash-double-dotted, total $(+\omega N)$ : solid line. The solid dots $(\bullet)$ are $2 \pi N$ partialwave cross sections, the open dots (०) are inelastic $\pi N \rightarrow \pi N$ partial-wave cross sections. For the data references, see Refs. ${ }^{1,2,3}$.

progress.

\section{Acknowledgments}

This work was supported by DFG and GSI Darmstadt.

\section{References}

1. G. Penner and U. Mosel, Phys. Rev. C66, 055211 (2002).

2. G. Penner and U. Mosel, Phys. Rev. C66, 055212 (2002).

3. G. Penner, Ph.D. dissertation (in English), Universität Giessen, September 2002, available via http://theorie.physik.uni-giessen.de .

4. K. Hagiwara et al. (Particle Data Group), Phys. Rev. D66, 010001 (2002).

5. T. Feuster and U. Mosel, Phys. Rev. C58, 457 (1998); ibid C59, 460 (1999).

6. G. Penner and U. Mosel, Phys. Rev. C65, 055202 (2002).

7. V. Shklyar, G. Penner, and U. Mosel, preprint hep-ph/0301152, submitted to Phys. Rev. C. 